\title{
Stock de carbono almacenado en la biomasa aérea, sotobosque y suelo en bosques andinos, Huancayo, Perú, 2013
}

Stock of Carbon stored in the biomass, sotobosque and soil in andean forests, Huancayo, Peru, 2013

Indira Luza Eyzaguirre

Universidad Continental, Prolg. Trujillo 684, Junín, Perú

DOI: https://doi.org/10.33017/RevECIPeru2014.0012/

\section{Resumen}

Uno de los grandes desafíos ambientales para la sociedad en la actualidad es el cambio climático, porque el incremento de sus indicadores contribuyen a la generación de los Gases de Efecto Invernadero, especialmente del dióxido de carbono $\left(\mathrm{CO}_{2}\right)$; por ello el análisis de la reserva o "Stock" de Carbono que almacenan los ecosistemas forestales como los bosques en sus diferentes componentes es de suma importancia; porque son considerados como los más importantes sumideros de carbono según los estudios de entidades de gran importancia como el Grupo Intergubernamental de Expertos sobre el Cambio Climático o IPCC por sus siglas en inglés. El objetivo principal de esta investigación fue: Comparar el Stock de Carbono almacenado en la biomasa aérea, sotobosque y suelo entre los bosques de Viques y la Hacienda Chorrillos y los objetivos específicos fueron: (i) Estimar dicho Stock en los componentes principales de estos ecosistemas forestales, (ii) Identificar la relación con los factores ambientales de la zona. La metodología utilizada fue no destructiva a través de la ecuación alométrica de Brown según el MINAM. Para la determinación de parcelas se usó sensoramiento remoto por medio del Google Earth obteniendo 6 y 4 parcelas generales, 1 para caracterización del suelo, 18 y 12 para análisis especial de Carbono/Nitrógeno, 6 y 4 para el inventario forestal en Viques y Chorrillos respectivamente. Se concluyó que el bosque de Viques tiene mayor stock de Carbono almacenado en sus componentes ( $33291 \mathrm{t} \mathrm{C/19ha} \mathrm{y} 122065$ t CO$/ 19 \mathrm{ha}$ ) a diferencia del bosque de Chorrillos (2934 t C/10ha y 10758 t CO $/ 10$ ha); esta variación se ve influenciado por la edad, hectáreas y factores ambientales (climáticos, topográficos, hidrográficos y tipo de ecorregión) comprobada estadísticamente con la prueba de hipótesis paramétrica t-student y no paramétrica de Wilcoxon para muestras independientes. La valoración económica de Viques fue de 332905 US\$/t C/19ha y para Chorrillos fue de 29339 US $\$ / t$ C/10ha.

Descriptores: Stock de Carbono, Cambio Climático, Ecosistema Forestal.

\section{Abstract}

One of the greatest environmental challenges today is the climate change, since the increase of the values of the indicators of climate change contribute to the generation of greenhouse gases, especially $\mathrm{CO}_{2}$; therefore the analysis of the reservation or "Stock" that store carbon forest ecosystems such as forests in its different components is paramount; since they are considered as the most important carbon sinks based on studies of major institutions such as the IPCC. The main objetive of this research was to compare the Stock Carbon stored in aboveground biomass, understory and ground between forests Viques and Finance Chorrillos and specific objectives were to estimate said Stock in the main components of these forest ecosystems, identify relation to environmental factors in the area. The methodology used was through nondestructive allometric equation Brown. For determination of remote sensing plots used by the Google Earth 6 and 4 general obtaining plots , 1 for soil characterization, 18 and 12 for special analysis of $\mathrm{C} / \mathrm{N}, 6$ and 4 for forest inventory and Chorrillos respectively Viques. This research concluded that the forest has more stock Viques Carbon stored in its 
components (33291, $122065 \mathrm{tnC} / 19 \mathrm{ha}$ and $\mathrm{tnCO}_{2} / 19 \mathrm{ha}$ ) unlike Chorrillos Forest (2934 and $10758 \mathrm{tnC} / 10 \mathrm{ha}$ $\operatorname{tnCO}_{2} / 10 \mathrm{ha}$ ) influenced by the age hectares and environmental factors (climatic, topographic, hydrographic and type of ecoregión) as parametric test $t$ - student hypothesis. Economic valuation of the first was U.S. \$ $332905 / \mathrm{tnC} / 19 \mathrm{ha}$ and the second 29339 U.S. $\$$ tnC/10ha. The ecosystem management is an approach to natural resource management that focuses on maintaining ecosystems to meet both ecological and human future needs. These multidisciplinary but interdisciplinary teams also are needed. Humans depend on ecosystems to meet our needs; likewise, ecosystems can be understood as the biophysical basis of the economy.

\section{Keywords: Stock Carbon, Climate Change, Forest Ecosystem}

\section{Introducción}

"Uno de los grandes desafíos ambientales en la actualidad es el cambio climático, ya que sus causas son locales con efectos globales. Más aún, el cambio climático es complejo ya que involucra muchos aspectos interconectados del sistema climático y sus impactos son múltiples y compuestos debido a las complejas estructuras sociales y económicas a nivel nacional como internacional"1.

La ubicación geográfica del Perú (franja ecuatorial) nos hace beneficiarios de tener una gran biodiversidad, pero actualmente los efectos de los problemas ambientales globales como el Cambio Climático vienen incrementándose a causa de las actividades antropogénicas diversas; es por eso que es necesario realizar investigaciones de corte científico en el campo de la Ingeniería Ambiental, teniendo en cuenta la realidad diversificada de los ecosistemas altoandinos, con el fin de probar la influencia de las actividades antropogénicas en la aceleración del Cambio Climático. El incremento de los valores de los indicadores del Cambio Climático contribuyen a la generación de los GEl, especialmente del $\mathrm{CO}_{2}$; por eso el análisis de la reserva o "Stock" de Carbono que almacenan los ecosistemas forestales como los bosques en sus diferentes componentes es de suma importancia; ya que son considerados como los más importantes sumideros de Carbono según los estudios de entidades de gran importancia como el IPCC. "Por ello la preocupación de la comunidad científica aumenta respecto al incremento de los espacios depredados, expansión de la agricultura industrializada" (ONU; 1992; p. 10), la tala de bosques, entre otros aspectos causados por las actividades antropogénicas que aceleran el proceso natural del Cambio Climático. La demanda del mercado económico está en expansión y causando efectos irreversibles en los sistemas de la Tierra; es por eso que se formaron acuerdos mundiales como el protocolo de Kioto donde se propusieron los Mecanismos de Desarrollo Limpio (MDL); el cual incluye el mercado o bonos de Carbono en el sector industrial dándole valor económico a un determinado ecosistema en base a las reservas o "Stock" de Carbono almacenado propuesto según el libro sobre Reducción de las Emisiones Producto de la Deforestación y la Degradación Ambiental o REDD por sus siglas en inglés. Los países en desarrollo aportan gran cantidad de biomasa vegetal que actúan a manera de pulmón por limpiar la atmósfera a través de la captación de $\mathrm{CO}_{2}$ en el proceso de fotosíntesis. Los bosques andinos son ecosistemas que están ubicados a más de 3.500 m.s.n.m. y son los guardianes de la vida en el planeta, los beneficios que proporcionan van del ámbito local al mundial. Los bosques andinos son claves para regular las actuales variaciones de temperatura, ya que crean microclimas. $^{2}$

El Valle del Mantaro se caracteriza por presentar variabilidad climática y topográfica de múltiples sectores de vegetación, las que reciben un impacto por las actividades antropogénicas que cada vez requiere de mayores extensiones de terreno altamente fértiles, es por eso que es necesario y urgente realizar una aproximación o estimación a la valoración económica de los servicios ambientales de ecosistemas forestales como los bosques; por ser una herramienta que brinda una perspectiva interdisciplinaria respecto a la reducción del Cambio Climático, principalmente de los GEl. En base a lo mencionado anteriormente se plantearon las siguientes interrogantes como problemas encontrados a las necesidades existentes:

${ }^{2}$ MINAM, 2011, p. 25

1 Takahashi, K.; 2010; p. 19. 
¿Cuál es la relación del Stock de Carbono almacenado en la biomasa aérea, sotobosque y suelo entre los bosques de Viques y la Hacienda Chorrillos?; ¿Cuánto es el Stock de Carbono almacenado en la biomasa aérea, sotobosque y suelo en los bosques de Viques y la Hacienda Chorrillos?; ¿Qué factores ambientales se relacionan con la variación del Stock de Carbono almacenado en la biomasa aérea, sotobosque y suelo en los bosques de Viques y la Hacienda Chorrillos?

El objetivo principal de esta investigación fue: Comparar el Stock de Carbono almacenado en la biomasa aérea, sotobosque y suelo entre los bosques de Viques y la Hacienda Chorrillos; asi mismo los específicos: Estimar el Stock de Carbono almacenado en la biomasa aérea, sotobosque y suelo en los bosques de Viques y la Hacienda Chorrillos; Identificar la relación que tienen los factores ambientales con la variación del Stock de Carbono almacenado en la biomasa aérea, sotobosque y suelo en los bosques de Viques y la Hacienda Chorrillos.

\section{Población y muestra:}

Las poblaciones estudiadas fueron los bosques andinos llamados: Viques y Hacienda Chorrillos ambos de especie predominante Eucalyptus globulus labill; estos bosques fueron seccionados por sensoramiento remoto para la posterior toma de muestras; en las siguientes figuras que se mostrarán a continuación se observa las especificaciones de cada zona estudiada:

Determinación de las muestras: Se parceló por sensoramiento remoto usando imágenes satelitales del Google Earth, como se observará en las Figs. 1 y 2 y las tablas 3 y 4 :

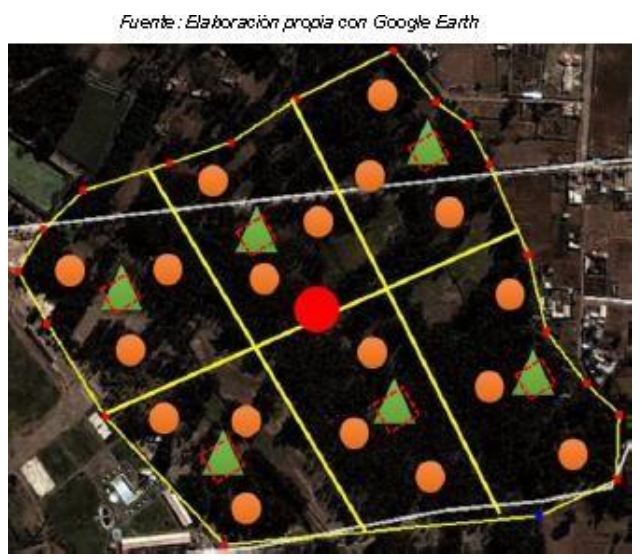

Figura 1: Muestras en el Bosque de Viques

Tabla 1: Muestras a recolectar en Viques Fuente: Elaboración propia.

\begin{tabular}{|c|c|c|}
\hline \multicolumn{2}{|l|}{ Bosque de "Vques" } & \multirow{2}{*}{ Simbolo } \\
\hline División de las parcelas & 6 & \\
\hline $\begin{array}{l}\text { Muestreo de suelo para análisis de } \\
\text { caracterización. }\end{array}$ & 1 & \\
\hline $\begin{array}{l}\text { Muestreo de suelo para análisis especial (C/N). } \\
\text { muestras desotobos que y muestr a de suelos para } \\
\text { densidad aparente. }\end{array}$ & 18 & \\
\hline $\begin{array}{l}\text { Inventario de árboles en subparcelas de } \\
20 \mathrm{~m} \times 20 \mathrm{~m} \text { (muestrarepresentatiou). }\end{array}$ & 6 & \\
\hline
\end{tabular}

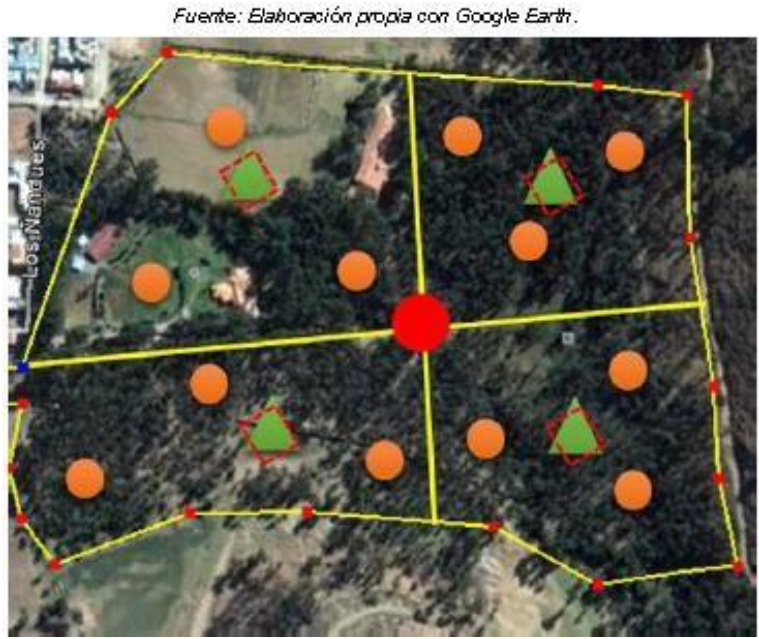

Figura 2: Muestras en el Bosque de Chorrillos

Tabla 2: Muestras a recolectar en Chorrillos Fuente: Elaboración propia.

\begin{tabular}{|l|c|c|}
\hline \multicolumn{2}{|c|}{ Bosque "Hacienda Chorrillos" } & \multirow{2}{*}{ Simbolo } \\
\cline { 1 - 2 } División de las parcelas & 4 & \\
\hline $\begin{array}{l}\text { Muestreo de suelo par a análisis de caracterización } \\
\text { sotobos que y muestra de suelos para densidad aparente }\end{array}$ & 12 & \\
\hline $\begin{array}{l}\text { Imventario de árboles en subp arcelas de } 10 \mathrm{~m} \times 10 \mathrm{~m} \text { (muestra } \\
\text { representativa) }\end{array}$ & 4 & \multirow{2}{*}{ S } \\
\hline
\end{tabular}

\section{Resultados y discusión}

\subsection{Climatología}

La variabilidad climática de ambas zonas estudiadas se evidencia en el análisis de la data obtenida con datos del Senamhi del año 20052009 como se muestra en la tabla (6): 
Tabla 3: Resultados de análisis referidos a la climatología.

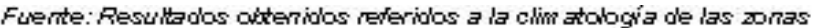
estudiadas.

\begin{tabular}{|c|c|c|c|}
\hline \multirow{4}{*}{ Área de estudio } & \multicolumn{3}{|c|}{ Cli matología } \\
\hline & Dbservacional & \multicolumn{2}{|c|}{ Däa } \\
\hline & \multirow[t]{2}{*}{ Vientos } & $\begin{array}{c}\text { Precipitación } \\
\text { Promedio }\end{array}$ & $\begin{array}{c}\text { Temperatura } \\
\text { Promedio }\end{array}$ \\
\hline & & mmiaño & वC. \\
\hline Estación en Viques & + & 15 & 21 \\
\hline $\begin{array}{c}\text { Estación en Huancayo } \\
\text { (más cer cana a la } \\
\text { Hacienda Chorrillos) }\end{array}$ & - & 63 & 19 \\
\hline
\end{tabular}

\subsection{Topografía}

Tabla 4: Caracteristicas topograficas de las zonas estudiadas.

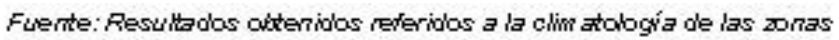
estudiadas.

\begin{tabular}{|c|c|c|c|c|c|c|c|}
\hline \multicolumn{8}{|c|}{ TOPOGRAFÍA } \\
\hline \multirow{2}{*}{$\begin{array}{l}\text { Area de } \\
\text { estudio }\end{array}$} & Cota 1 & $\operatorname{cdt}=2$ & \multirow{2}{*}{$\begin{array}{c}\text { Dif erencia } \\
\text { de cotas } \\
h\end{array}$} & \multicolumn{2}{|c|}{ Longitud } & \multicolumn{2}{|c|}{ Pendiente $(\% /$ ) } \\
\hline & \multicolumn{2}{|c|}{ msn.m. } & & $\mathrm{km}$ & $m$ & \multicolumn{2}{|c|}{ SA, /L } \\
\hline Viques & 3185 & 3161 & 24 & 0.5 & 530 & 0.05 & $5 \%$ \\
\hline $\begin{array}{l}\text { Hacienda } \\
\text { Chorrillos }\end{array}$ & 3414 & 3356 & 58 & 0.4 & 390 & 0.15 & $15 \%$ \\
\hline
\end{tabular}

Como se evidencia en la Fig. 3, el bosque de Viques tiene una superficie plana sin presencia de lomas, ya que se encuentra a una elevación de 3171 m.s.n.m. presentando mayor presencia de vientos a diferencia de la Fig. 4 donde se muestra el bosque Hacieda Chorrillos; este útimo por estar a una elevación de 3388 m.s.n.m. cuenta con menor presencia de vientos; este factor topográfico influye en la variabilidad climática y fisiológica de sus componentes por lo tanto en la captura de carbono de ambos bosques.

Fuente: in ágenes Szalitzales analizadas con Google Eath
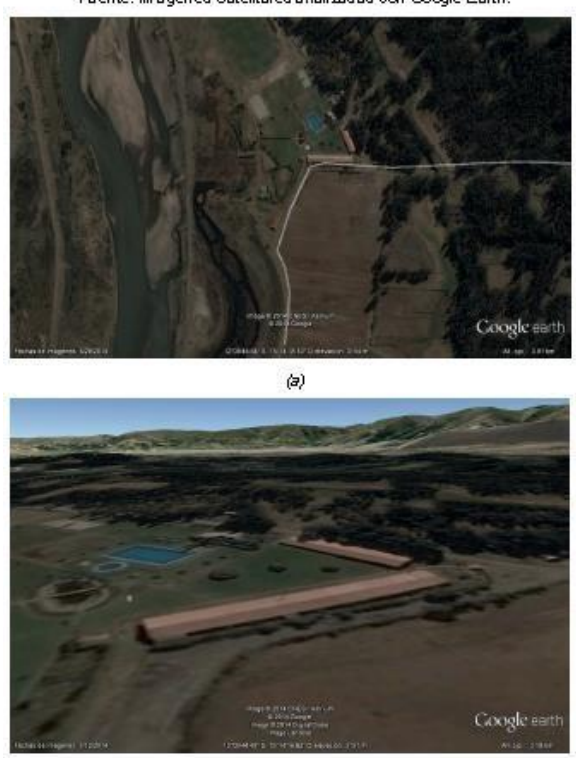

Figura 3: Superficie topografica del bosque de Viques $(a ; b)$
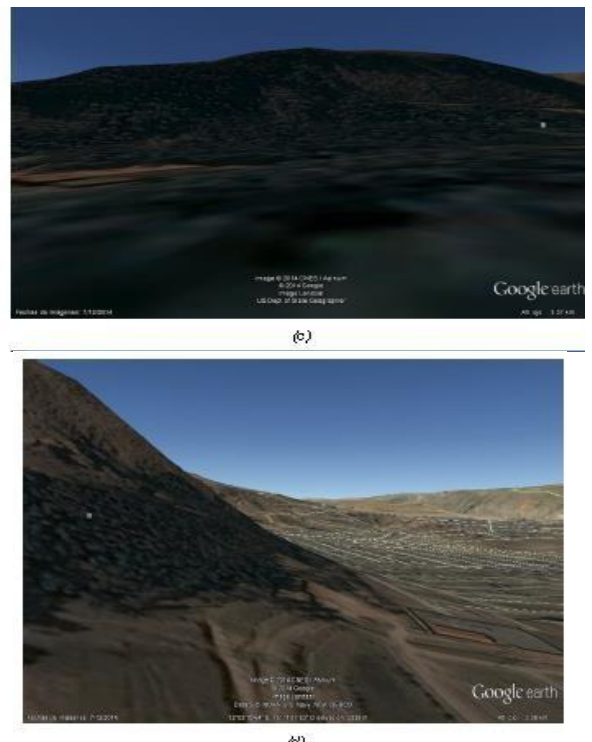

(i)

Figura 4: Superficie topografica del bosque de Chorrillos (c; d)

\subsection{Hidrografía}

Como se evidencia en la tabla 8 se identificó (observacinal) un cuerpo de agua superficie principal cerca al bosque de Viques que es el Río Mantaro; así mismo se observó varios cuerpos superficiales de agua (riachuelos) cerca del bosque de Chorrillos, pero cerca de este último se encuentra el Nevado del Huaytapallana, que influye indirectamente en la variabilidad climática de este bosque; esta información se corrobora con los resultados del análisis de suelos donde hay mayor porcentaje de humedad en los suelos del bosque Chorrillos (101\%) que en el de Viques (1\%); ya que el ciclo hidrológico se relaciona con la litósfera y por ello con la biósfera de las zonas estudiadas.

Tabla 5: Resultados de la hidrografia.

Fuente: Resultados oistenidos reteridos a la hidrogratia de las zonas estudiadas.

\begin{tabular}{|c|c|}
\hline & HIDRDGRAFIA \\
\hline Area de estudio & Hidográia \\
\hline Viques & Rio Mantaro \\
\hline $\begin{array}{c}\text { Hacienda } \\
\text { Chorrillos }\end{array}$ & Rio Shulleas y Riachuelos. Nevado del \\
Huaytapallana.
\end{tabular}
Takla 39: Rescltados oixenidos de la Aolrograła.

\subsection{Ecorregión}




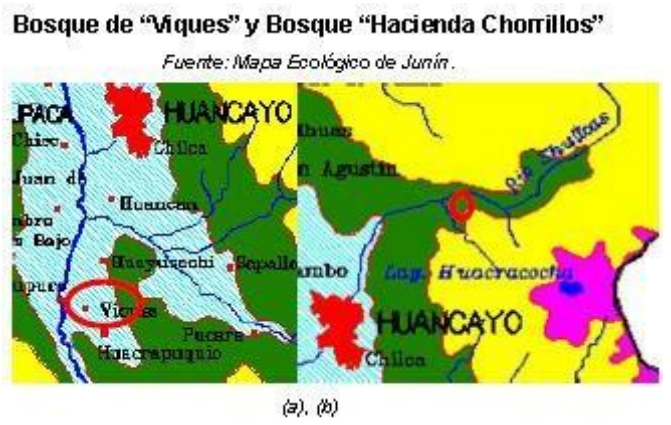

Figura 5: Tipo de bosques (a) Bosque SecoMontano Bajo Tropical; (b) Bosque HumedoMontano Tropical.

\subsection{Biomasa aérea}

La edad de los bosques estudiados se ven reflejados en el promedio del DAP (diámetro a la altura de pecho) que fue $17 \mathrm{~cm}$ y $23 \mathrm{~cm}$ para el bosque de Viques $y$ Hacienda Chorrillos respectivamente como se muestra en la tabla (9), así mismo, estos valores indican el nivel de maduración de la especie estudiada ya que está directamente relacionada con la cantidad de la reserva o Stock de Carbono que almacena.

\section{Tabla 6: Resultados del inventario forestal.}

Fuente: Resultados obtenidos referidos al número de árboles inventariados, promedio DAP, años y hectáreas de cada bosque.

\begin{tabular}{|c|c|c|c|c|}
\hline LUGAR & $\begin{array}{c}\text { \#árboles } \\
\text { inventariados }\end{array}$ & $\begin{array}{c}\text { Promedio de } \\
\text { DAP (cm) }\end{array}$ & Edad (años) & ha \\
\hline VIQUES & 1085 & 17 & 10 & 19 \\
\hline CHORRILLOS & 232 & 23 & 10 & 10 \\
\hline
\end{tabular}

Existe una explicación fisiológica para que haya una variación en el Stock de Carbono respecto a la edad de los bosques (Organero, E.; Gimeno, J.; 2007). Algunas investigaciones mencionan que el órgano principal de un árbol es la raíz, porque se encarga de anclar la planta al sustrato, absorbe agua, sales minerales pero también carbono del suelo conduciéndolos por medio del xilema y floema acumulando progresivamente esta sustancia como reserva y como todo ciclo, el árbol cambia sus hojas cada cierto tiempo dejándolas caer sobre el suelo, sirviendo así como cobertura y materia orgánica al suelo, así mismo se bioacumula en dicho componente y el ciclo continúa (Benito, Aparacio, Garzón y Zavala.

España).

El principal proceso en la reserva de carbono es la Fotosíntesis, ya que a través de la estructura cloroplastos y clorofila que es una molécula que tiene la función de capturar a través del mecanismo de carboxilación a partir del $\mathrm{CO}_{2}$ atmosférico por medio del ciclo de Calvin y Benson o ciclo C3; dando lugar al Ciclo biogeoquímico del Carbono el cual está influenciado por factores ambientales como la temperatura, radiación, precipitación y humedad.

Por ello en los resultados de la tabla 9 se aprecia la diferencia del promedio de DAP en $\mathrm{cm}$ de los árboles inventariados siendo en Viques 1085 con una superficie de 19 ha a diferencia de Chorrillos que fue 232 árboles con una superficie de 10 ha; ambos bosques tienen la misma edad, la diferencia del diámetro de los árboles inventariados radica en el cuidado de cada bosque; mientras que el bosque de Viques está destinado para el uso forestal por ello los árboles son relativamente jóvenes, ya que son reemplazados por otros cada cierto tiempo; a diferencia de Chorrillos donde se aprovecha uno de los servicios ambientlales del bosque que es la belleza escénica; por ello se encontró arboles más maduros en cuanto a biomasa se trata. Esta afirmación se recolectó de las entrevistas informales que se realizó a los pobladores de cada bosque, así como se muestra en la Fig. 7.

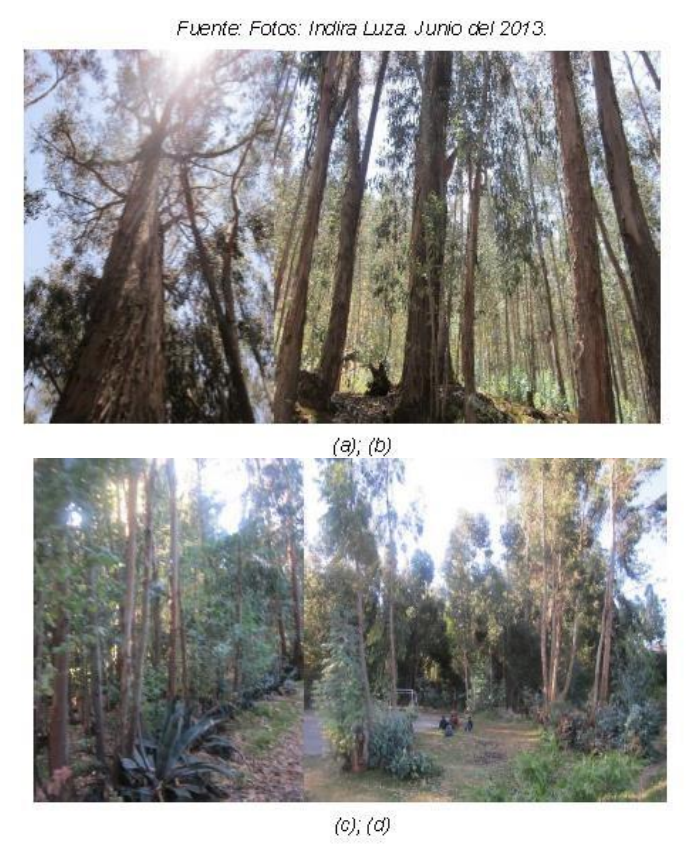

Figura 6: (a; b) Vista de árboles más maduros en el boque de Chorrillos. ( $c$ y d) Vista de los árboles más delgados en cuanto a diámetro del bosque de Viques. 
La vegetación arbórea supone el $70 \%$ del carbono acumulado en la vegetación mundial, este valor se asemeja al resultado final obtenido como se muestra en la tabla 10; donde el $\mathrm{CO}_{2}(100 \%)$ fue 91794 t $\mathrm{CO}_{2} / 19$ ha y $7417 \mathrm{t} \mathrm{CO}_{2} / 10$ ha para Viques y Chorrillos respectivamente siendo el $86 \%$ y $36 \%$ del total la biomasa aérea en ambos bosques. El stock de Carbono se correlaciona con variables climáticas y estructurales de cada parcela aunque cada tipo de bosque se comporta de forma diferente, es por eso que según la revisión bibliográfica y los resultados obtenidos los factores ambientales influyen directamente en el stock de carbono de cada bosque.

Tabla 7: Resultados de la estimación en la biomasa aérea.

\begin{tabular}{|c|c|c|c|}
\hline \multirow{2}{*}{$\begin{array}{c}\text { COMPONENTE } \\
\text { ZONA DE ESTUDIO }\end{array}$} & \multicolumn{3}{|c|}{$\begin{array}{l}\text { BIOMASA AÉREA TOTAL (árboles y } \\
\text { raices) }\end{array}$} \\
\hline & tha total & t C/ha total & $\begin{array}{l}\mathrm{t} \mathrm{CO}_{2} \mathrm{ha} \\
\text { total }\end{array}$ \\
\hline VIQUES (19ha) & 66192 & 29787 & 109217 \\
\hline CHORRILLOS (10ha) & 3647 & 1641 & 6018 \\
\hline
\end{tabular}

\subsection{Sotobosque}

La estimación de la biomasa para el componente de SOTOBOSQUE se hizo a través del método de humedad como se muestra en la tabla 11:

Tabla 8: Resultados de la estimación en el Sotobosque.

Fuente: Resultados de la variación en el Sotobosque.

\begin{tabular}{|c|c|c|c|}
\hline \multirow{2}{*}{ zONA DE ESTUDIO } & \multicolumn{3}{|c|}{ SOTOBOSQUE } \\
\cline { 2 - 4 } & tha total & t C/ha total & t co /ha total \\
\hline VIQUES (19ha) & 29 & 15 & 54 \\
\hline CHORRILLOS (10ha) & 116 & 58 & 213 \\
\hline
\end{tabular}

Existe una influencia de la precipitación (lluvias todo el año) en la producción del sotobosque, concluyéndose que los bosques absorben, almacenan y liberan alrededor de 1146 millones de toneladas de carbono donde el $37 \%$ se encuentra en bajas latitudes, $14 \%$ en latitudes medias y $49 \%$ en latitudes altas. Por lo tanto los resultados se vieron influenciados por factores climáticos, pero también por la dinámica de cada bosque; en Viques se observó diferentes escenarios algunos con vegetación primaria, escenarios pedregosos y sin presencia de sotobosque; a diferencia de Chorrillos donde se observó zonas con mayor porcentaje de sotobosque como se muestra en la
Fig. 8.
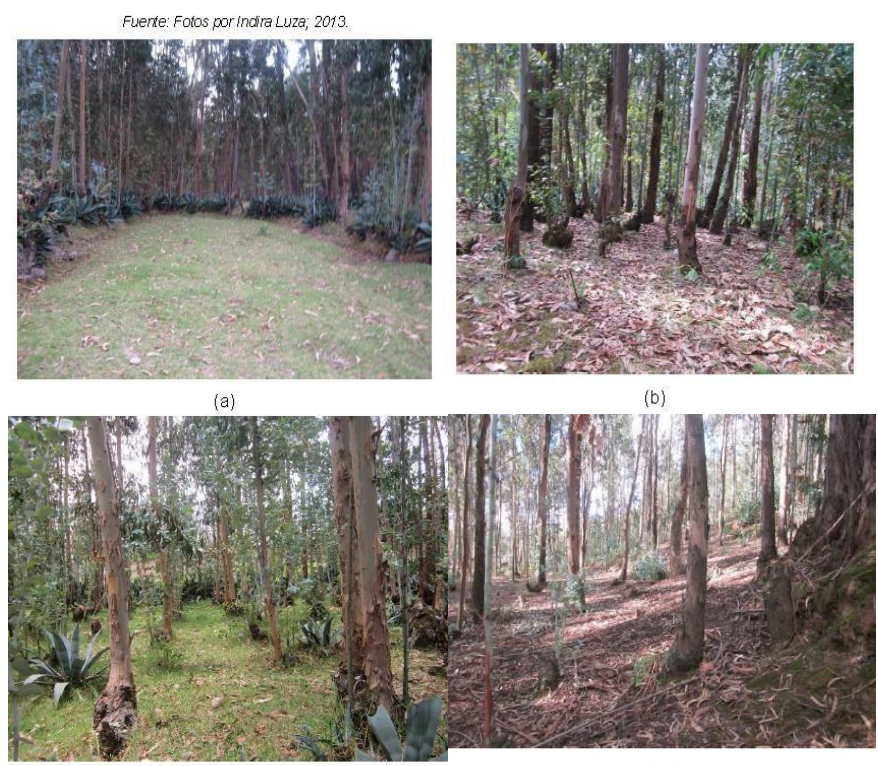

(c)

(d)

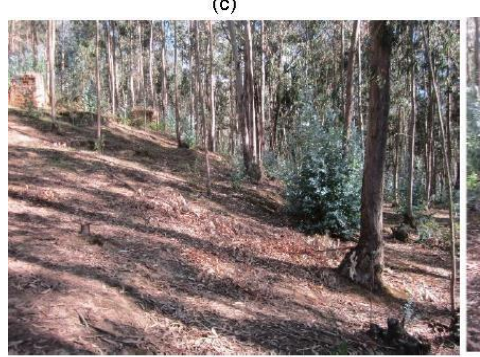

(e)

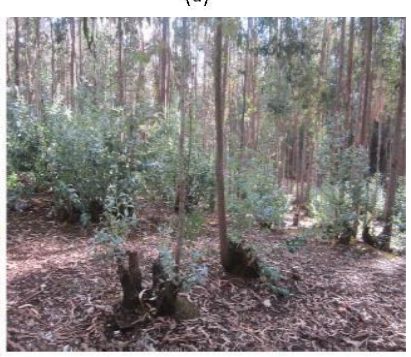

(f)
Figura 7: Bosque de Viques (a) escenario con ausencia de sotobosque, (b) escenario con sotobosque y (c) escenario pedregoso y presencia de especies de zonas áridas. Bosque de Chorrillos: (d, e y f) todos los escenarios con presencia de sotobosque.

\subsection{Suelo}

Se muestra en la tabla 12 los resultados de densidad aparente $\left(\mathrm{g} / \mathrm{cm}^{3}\right)$, así mismo en la tabla 13 se muestra los resultados de la temperatura del suelo:

Tabla 9: Resultados de densidad aparente, temperatura promedio y porcentaje de humedad en el suelo.

Fuente: Resultados de la variación en porcentaje en los subcomponentes Hojas, Tallos y Otros en Viques y chorrillos.

\begin{tabular}{|c|c|c|c|}
\hline \multirow{2}{*}{ ZONA DE ESTUDIO } & \multicolumn{3}{|c|}{ Propiedades } \\
\cline { 2 - 4 } & $\begin{array}{c}\text { Densidad } \\
\text { aparente }\end{array}$ & $\begin{array}{c}\text { Temperatura } \\
\text { promedio }\end{array}$ & \%Humedad \\
\cline { 2 - 4 } & \multirow{2}{*}{$\mathbf{g} / \mathbf{c m}^{\mathbf{3}}$} & $\mathbf{C}^{\mathbf{0}}$ & \% \\
\cline { 3 - 4 } & 0.94 & 19.09 & 0.08 \\
\hline VIQUES (19ha) & 1.27 & 17.72 & 8.38 \\
\hline CHORRILLOS (10ha) & &
\end{tabular}

El contenido en porcentaje de $\mathrm{C}$ es un reservorio importante para la fijación de $\mathrm{C}$ atmosférico (Sánchez, 2010). Ante ello la relación C/N esta 
relación es un indicador de la acumulación contínua de $\mathrm{MO}$ en los suelos. El almacenamiento de $\mathrm{C}$ y $\mathrm{N}$ en el suelo varía de acuerdo con la altura sobre el nivel del mar. Se encontró mayor cantidad de estos componentes entre los $0-10 \mathrm{~cm}$ del suelo. La abundancia del $\mathrm{C}$ y $\mathrm{N}$ dependen del tipo de uso del suelo, la cobertura vegetal y la fijación de los nutrientes (Carvajal, 2008).

Tabla 10: Resultados del análisis de caracterización del suelo según el laboratorio de la UNALM.

\begin{tabular}{|c|c|c|c|c|c|c|c|c|}
\hline \multirow{3}{*}{$\begin{array}{l}\text { ZONA DE } \\
\text { ESTUDIO }\end{array}$} & \multicolumn{8}{|c|}{ PROPIEDADES } \\
\hline & $\mathrm{pH}$ & \multicolumn{3}{|c|}{$\begin{array}{l}\text { Conductividad } \\
\text { Eléctrica }\end{array}$} & $\% \mathrm{C}$ & $\% \mathrm{~N}$ & \multicolumn{2}{|c|}{ Relación } \\
\hline & Tabla & \multicolumn{3}{|c|}{$\mathrm{dS} / \mathrm{m}$} & $\%$ & $\%$ & \\
\hline VIQUES (19ha) & 8.32 & & 09 & & \begin{tabular}{l|l}
37.81 & 3 \\
3
\end{tabular} & 3.40 & \multicolumn{2}{|l|}{199.64} \\
\hline $\begin{array}{l}\text { CHORRILLOS } \\
\text { (10ha) }\end{array}$ & 5.53 & \multicolumn{3}{|c|}{0.45} & $19.09 \quad 1$ & 1.63 & \multicolumn{2}{|l|}{141.76} \\
\hline \multirow{3}{*}{$\begin{array}{l}\text { ZONA DE } \\
\text { ESTUDIO }\end{array}$} & \multicolumn{8}{|c|}{ PROPIEDADES } \\
\hline & $\begin{array}{l}\text { MATERIA } \\
\text { ORGANICA }\end{array}$ & $\mathrm{CaCO}_{3}$ & $P$ & K & \multirow{2}{*}{$\begin{array}{l}\text { Clase } \\
\text { Textural }\end{array}$} & \multicolumn{3}{|c|}{ Análisis Mecánico } \\
\hline & $\%$ & $\%$ & $\mathrm{ppm}$ & $\mathrm{ppm}$ & & $\begin{array}{l}\text { Are } \\
\%\end{array}$ & \begin{tabular}{l|l|} 
a & Limo \\
$\%$
\end{tabular} & Arcilla \\
\hline $\begin{array}{l}\text { VIQUES } \\
\text { (19ha) }\end{array}$ & 2.77 & 8.10 & 2.7 & 331 & Fr.A. & 57 & 24 & 19 \\
\hline $\begin{array}{c}\text { CHORRILLOS } \\
\text { (10ha) }\end{array}$ & 1.38 & 0.00 & 3.9 & 117 & Fr. & 41 & 40 & 19 \\
\hline
\end{tabular}

En la evaluación de parámetros físico-químicos como la temperatura, $\mathrm{pH}$, disponibilidad de oxígeno, nutrientes inorgánicos, clase tectural, entre otros influyen en la descomposición de la materia orgánica y así en las reservas de carbono en el componente suelo.

\section{Disminución de la pérdida de carbono}

Aparte de los factores climáticos principalmente la temperatura los procesos más importantes que causan pérdidas de carbono del suelo son la erosión y la mineralización de la materia orgánica. La captura de Carbono y el aumento de la MO del suelo tendrán un impacto directo sobre la calidad y la fertilidad de los suelos. En lo que se refiere al ciclo del carbono, habrá una mayor captura de carbono por la biomasa aérea y un correlativo ingreso de carbono en el suelo a partir de los residuos de las plantas y del crecimiento y la muerte de las raíces más finas. Habrá una mayor captura de carbono por la biomasa aérea y un correlativo ingreso de carbono en el suelo a partir de los residuos de las plantas y del crecimiento y la muerte de las raíces más finas.

En la Fig. 11 se muestra la presencia de Líquenes en ambos bosques, ya sea como hongos o musgos; debido a que el Eucalipto brinda las condiciones (humedad) para que estos seres bióticos vivan. En diversos estudios se evidenció que los líquenes tienen un importante papel como bioindicadores de lectura inmediata de contaminación medioambiental, de los cambios climáticos y de la estabilización del suelo.

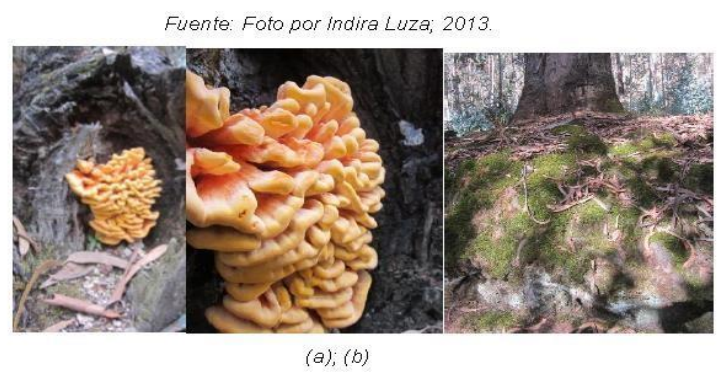

Figura 8: Vista de los líquenes presentes en los árboles de Viques (a) y (b) musgos en Chorrillos
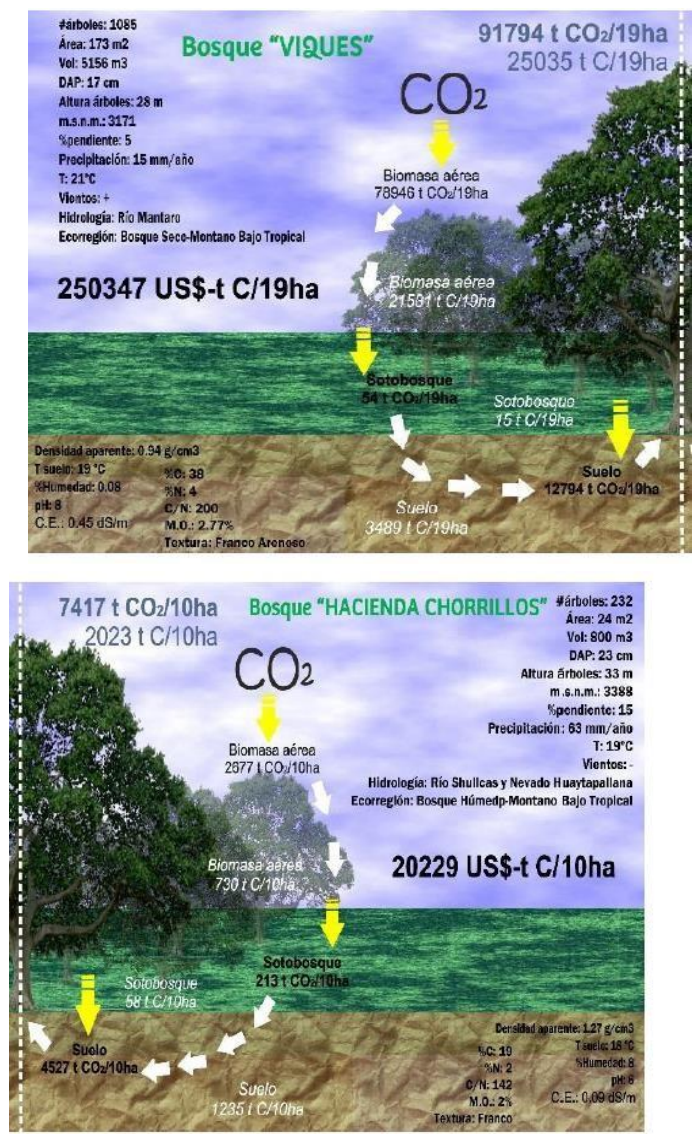

Figura 9: Flujo del stock de carbono y otros componentes analizados en los ecosistemas forestales.

Los líquenes son asociaciones mutualistas entre hongos y algas o cianobacterias que pueden funcionar en la naturaleza como una unidad. Las razones por las cuales se usan los líquenes como bioindicadores, biomonitores y bioacumuladores es que son de naturaleza simbiótica, es decir si uno de ellos muere porque son sensibles a 
pequeños cambios el otro también se ve afectado, así mismo porque obtienen la mayor parte de sus nutrientes del aire, lo que los hace muy sensibles a las impurezas del medio. En conclusión, son empleados para conocer la calidad del ambiente terrestre y atmosférico. (Mendes y Monge; 2011)

\section{Conclusiones}

Se comparó exitosamente el stock de Carbono almacenado en la biomasa aérea, sotobosque y suelo entre los bosques de Viques y Chorrillos, por lo tanto se concluye que el bosque de Viques almacena en total 25035 t C/19ha y 91794 t CO$_{2} / 19$ ha siendo mayor que Chorrillos con 2023 t C/10ha y 7417 t $\mathrm{CO}_{2} / 10$ ha. Esta diferencia comprobada a través de la prueba de hipótesis se debe a la influencia de los factores ambientales como la variabilidad climática, topográfica, hidrográfica y tipo de ecorregión, así mismo el área total y años de cada ecosistema forestal; siendo Viques de mayores hectáreas (19) a diferencia de Chorrillos (10). Ambos bosques son de especie Eucaypto siendo esta una de las especies que mayor carbono captura. Esta conclusión se evidencia en la base de datos obtenida de ambos bosques, especialmente la diferencia en el relieve topográfico; Viques tiene un terreno con menor pendiente $(5 \%)$ y más árido $(0.08 \%$ humedad) que el de Chorrillos que se encuentra en un terreno de influencia hídrica directa (escorrentía e infiltraciones), con mayor pendiente (15\%) y de suelos más húmedos ( $8 \%$ de humedad).

Se estimó en los tres componentes principales las reservas de carbono, obteniendo en Viques un total de biomasa (árboles y raíces) de 47846 t/19ha, 21531 t C/19ha y 78946 t CO$_{2} / 19$ ha a diferencia de Chorrillos con un total de $1623 \mathrm{t} / 10 \mathrm{ha}, 730 \mathrm{t} \mathrm{C} / 10 \mathrm{ha}$ y $2677 \mathrm{t} \mathrm{CO}_{2} / 10 \mathrm{ha}$ en el mismo componente. Esta diferencia se debe a que Viques tiene mayores hectáreas y por lo tanto mayor cantidad de individuos arbóreos por ello el inventario de este bosque fue en total de 1085 árboles a diferencia de Chorrillos que fue de solo 232 árboles.

La estimación del componente Sotobosque también mostró una diferencia para Viques con un 29 t/19ha, $15 \mathrm{t} \mathrm{C} / 19$ ha y $54 \mathrm{t} \mathrm{CO}_{2} / 19$ ha a diferencia de Chorrillos con un 116 t/10ha, 58 t C/10ha y 213 t CO $/ 10 \mathrm{ha}$; evidenciándose que el primer bosque tuvo menor producción de este componente el tiempo de recolección de muestras y Chorrillos tuvo más producción primaria neta de este componente; así mismo porque en el primer bosque hay mayor presencia de vientos y esto hace que las hojas caídas se esparzan y se desplacen a diferencia de Chorrillos el cual presenta una topografía más cerrada sin mucha presencia de vientos haciendo que se acumule más las hojas.

La estimación del último componente: Suelo fuede un total de $3489 \mathrm{t} \mathrm{C/19ha} \mathrm{y} 12794 \mathrm{t} \mathrm{CO}_{2} / 19$ ha para Viques y de un total de $1235 \mathrm{t} \mathrm{C} / 10$ y $4527 \mathrm{t}$ $\mathrm{CO}_{2} / 10$ ha para Chorrillos. Esta diferencia se debe a que los suelos de Viques presentan mayor porcentaje de Materia orgánica (2.77) que está relacionada con el porcentaje de Nitrógeno (3.40), Carbono (37.81) según el indicador de $\mathrm{pH}$ que es básico (8.32) dando las condiciones para elevar los parámetros mencionados anteriormente; esto a diferencia de Chorrillos que presenta menor porcentaje de Materia Orgánica (1.38) al igual que los porcentajes de Nitrógeno (1.63) y Carbono (19.09) con un pH más ácido (5.53). estos parámetros físicos - químicos mencionados influyen directamente en la producción de materia orgánica que es el principal componente del flujo de Carbono y de la captura de $\mathrm{CO}_{2}$.

La gestión de los ecosistemas es un enfoque para la gestión de recursos naturales porque se centra en el mantenimiento de los ecosistemas para satisfacer las necesidades futuras tanto ecológicas como humanas. Para ello se necesitan equipos multidisciplinarios, pero además interdisciplinarios; ya que los árboles son complejos Ecosistémicos de seres vivos siendo los bosques andinos los más altos del mundo y se extienden en las cimas y los valles interandinos de muchos departamentos del Perú (Junín, Huánuco, Huancavelica, Ayacucho, Apurímac, Cajamarca y Cusco). Están entre los 2000 - 4000 m.s.n.m.

Los seres humanos dependemos de los ecosistemas para satisfacer nuestras necesidades; así mismo los ecosistemas pueden ser entendidos como las bases biofísicas de la economía. Los servicios de los ecosistemas o servicios ambientales han sido definidos como los beneficios que los humanos obtenemos de los ecosistemas. La clasificación de los ecosistemas más utilizados es la aportada por la Evaluación de Ecosistemas del Milenio, iniciativa de las Naciones Unidad. El informe final de dicha evaluación concluye que el $60 \%$ de los servicios de los ecosistemas a escala global están en declive o están siendo utilizados de forma insostenible. 


\section{Agradecimientos}

Agradezco todas las maravillosas enseñanzas de toda la experiencia y conocimiento transmitido de las personas más geniales que haya conocido: Ing. Luis Suarez, Dr. Jorge Ferrer, Dr. Francisco Tarazona, Ing. Guillermo Jaramillo, Mg. David Pizarro, Lic. Wilmer Medina, Ing. Olga Kostenko, Lic. Luz Supo. A mis grandes amigos Trilce Guerra, Naty Huari, Julio Capacyachi, Daryl Estrella, Gaby Buendía, Janet Baltazar, Daniel Asorza, Joseph Sedano, Alfonso Márquez, Santiago y Yanina Escobar por hacer de mis días en la universidad tan divertidas y por los únicos y especiales momentos compartidos y a la memoria de mi queridímo amigo José Miguel Dominguez por ayudarme tanto en las salidas de campo.

\section{Referencias}

[1] A. Alvarado, R. Forsythe, Variación de la densidad aparente en órdenes de suelos de Costa Rica, Costa Rica, 2005.

[2] T. Barker, O. Phillips y J. Lloyd, Análisis del stock y flujos de carbono, una herramienta para los proyectos REDD. Iquitos, Perú, University of Leeds, UK, 2007.

[3] A. Brack, Enciclopedia temática, Ecología, Perú, 2009.

[4] A. Brack y C. Mendiola, Ecología del Perú. Perú, 2000.

[5] C. Chambi, Valoración económica de secuestro de carbono mediante la simulación aplicada a la zona boscosa del rio Inambari y Madre de Dios. IICFOE, Perú, 2001.

[6] V. Dávila, F. Retamozo, L. Suarez, Almacenamiento de carbono y flujo de $\mathrm{CO} 2$ en los suelos con plantaciones de tres especies forestales en el Valle del Mantaro. Tesis para optar el título de Ingeniero Forestal, UNCP, 2008.

[7] Ecosystem Marketplace, Getting Started, un manual introductorio para evaluar y desarrollar pagos por servicios ambientales, 2007.

[8] FAO, Informes sobre recursos mundiales de suelos. Captura de carbono en los suelos para un mejor manejo de la tierra, Francia, 2002.

[9] I. Hawksworth, Líquenes como bioindicadores inmediatos de contaminación y cambios medioambientales en los trópicos, España, 2000.

[10] C. Martel, Cuantificación del carbono almacenado en formaciones vegetales amazónicas en "CICRA". UNALM. Madre de Dios. Perú, 2012.
[11] MINAM, Segunda comunicación nacional del Perú a la CMNUCC. Identificación de metodologías existentes para determinar stock de carbono en ecosistemas forestales. Perú, 2009.

[12] E. Odum, y G. Barret, Fundamentos de la ecología. México, 2008.

[13] O. Phillips, J. Lloyd, Análisis del stock y flujos de carbón, una herramienta para los proyectos REDD. Tim Barker University of Leeds. Iquitos, Perú, 2007.

[14] PNUMA, Protocolo de Kioto de la Convención Marco De Las Naciones Unidas Sobre El Cambio Climático. Japón, 1998.

[15] K. Takahashi, Cambio climático, investigación e incertidumbre. Instituto Geofísico del Perú IGP. Perú, 2010.

E-mail: Indiraluza@gmail.com 